\title{
AN EULER-POISSON SYSTEM IN PLASMAS
}

\author{
A. NOURI ${ }^{1}$
}

(Received 4 October 1995)

\begin{abstract}
A local existence and uniqueness result is proved for the three-dimensional Euler-Poisson system without a pressure term which arises in plasma physics.
\end{abstract}

\section{Introduction}

For some non-viscous fluids occurring in plasma physics, pressure effects are negligible with respect to charge effects. In these cases, it is physically relevant to set the pressure to be constant. The plasma is then properly defined by the Euler-Poisson system:

$$
\begin{aligned}
& \frac{\partial \rho}{\partial t}+\nabla_{x} \cdot(\rho U)=0, \\
& \frac{\partial(\rho U)}{\partial t}+\nabla_{x} \cdot(\rho U \otimes U)=\rho E,
\end{aligned}
$$

with the initial conditions

$$
\rho(t=0)=\rho_{0}, \quad U(t=0)=U_{0} .
$$

Here $x$ and $t$ are the physical space and time coordinates, $\rho$ is the density, $U$ is the fluid velocity and $E$ is the electric field. Equations (1.1) and (1.2) describe the conservation of mass and momentum respectively. The electric field $E$ is given by the elliptic-type Poisson equation in free space

$$
E=-\nabla_{x} \Phi, \quad-\Delta_{x} \Phi=4 \pi \rho,
$$

with $\Phi(t, x)$ tending to 0 as $|x|$ tends to $\infty$. In fact, in discussing solutions of (1.1)(1.3) in regions of smoothness for $U$, one often uses the density and the velocity as

\footnotetext{
${ }^{1}$ Insa, 20 av A Einstein, 69621 Villeurbanne Cedex, France.

(C) Australian Mathematical Society 2000, Serial-fee code $0334-2700 / 00$
} 
independent variables. In regions of smoothness, the equations can be equivalently written in terms of the variables $\rho$ and $U$ :

$$
\begin{aligned}
& \frac{D \rho}{D t}=-\rho \nabla_{x} \cdot U, \\
& \frac{D U}{D t}=E,
\end{aligned}
$$

where

$$
\frac{D}{D t}=\frac{\partial}{\partial t}+U_{i} \frac{\partial}{\partial x_{i}}
$$

is the convective derivative along the fluid flow. In the case of gas dynamics in onedimensional space, which leads to similar equations without the source term, Eulerian and Lagrangian formulations have been proved to be equivalent within a specific class of weak bounded measurable functions [10]. Moreover the one-dimensional Cauchy problem associated with (1.1)-(1.3) without any coupling with Poisson's equation (1.4) has been considered via two different approaches. Measure solutions defined for all positive times are developed in [1] for two specific types of initial data, a system of particles and the Riemann problem. Multivalued solutions, generalizing the usual notion of weak $L^{\infty}$ solutions, are produced in [3], and numerically studied, including Poisson's equation, in [2]. Here the problem is set in three-dimensional space. We consider smooth solutions for the system of gas dynamics without a pressure term coupled with Poisson's equation. For smooth solutions, local existence must be considered. Equations (1.1)-(1.3) can be viewed as a Cauchy problem of the form

$$
V_{t}+A_{j}(V) V_{x_{j}}=B(t, x, V), \quad V(t=0)=V_{0},
$$

where $V=(\rho, U)$. This does not match the common structure of Friedrich's symmetric systems, for which there is a basic local existence theorem of smooth solutions [7]. Indeed, system (1.8) is not hyperbolic since no linear combination of $A_{j}(V)$ is diagonalizable. In this paper we obtain a local existence and uniqueness theorem for an arbitrary large initial concentration and velocities in three-dimensional space. We generalize a previous result (see [8]) obtained in studying a paraxial approximation in a one-dimensional frame. The key points of the proof are a Lagrangian approach allowed by the non-generic type of the studied PDE system, together with control of the density and its first derivatives. We now state the main result of this paper.

THEOREM 1.1. Let $r>3$. Let $\rho_{0}$ be a function with a compact support $K$, belonging to $W^{1, \infty}(K)$ and let $u_{0}, v_{0}$ and $w_{0}$ be functions of $W^{1, \infty}\left(\mathbb{R}^{3}\right)$ with second-order derivatives belonging to $L^{r}\left(\mathbb{B}^{3}\right)$. Then there exists a time $T$ such that the Cauchy 
problem (1.1)-(1.4) has a solution $(\rho, u, v, w, E)$ where

$$
\begin{aligned}
\rho & \in L^{\infty}\left((0, T) \times \mathbb{R}^{3}\right) \cap W^{1, r}\left((0, T) \times \mathbb{R}^{3}\right), \\
(u, v, w) & \in\left(W^{1, \infty}\left((0, T) \times \mathbb{R}^{3}\right)\right)^{3}, \\
E & \in W^{2, r}\left((0, T) \times \mathbb{R}^{3}\right) .
\end{aligned}
$$

Moreover $\rho$ is compactly supported and the solution is unique in the class of functions $(\rho, u, v, w)$ such that the second-order derivatives of $u, v$ and $w$ belong to $L^{r}((0, T) \times$ $\left.\mathbb{R}^{3}\right)$.

\section{Preliminary results}

It is well known that up to a multiplicative constant set equal to 1 the solution of Poisson's equation (1.4) in $\mathbb{R}^{3}$ is

$$
\begin{aligned}
E_{1} & =E_{1}(\rho)(t, x, y, z) \\
& =\int_{\mathbb{R}^{3}} \frac{x-x^{\prime}}{\left(x-x^{\prime}\right)^{2}+\left(y-y^{\prime}\right)^{2}+\left(z-z^{\prime}\right)^{2}} \rho\left(t, x^{\prime}, y^{\prime}, z^{\prime}\right) d x^{\prime} d y^{\prime} d z^{\prime}, \\
E_{2} & =E_{2}(\rho)(t, x, y, z) \\
& =\int_{\mathbb{R}^{3}} \frac{y-y^{\prime}}{\left(x-x^{\prime}\right)^{2}+\left(y-y^{\prime}\right)^{2}+\left(z-z^{\prime}\right)^{2}} \rho\left(t, x^{\prime}, y^{\prime}, z^{\prime}\right) d x^{\prime} d y^{\prime} d z^{\prime}, \\
E_{3} & =E_{3}(\rho)(t, x, y, z) \\
& =\int_{\mathbb{R}}^{3} \frac{z-z^{\prime}}{\left(x-x^{\prime}\right)^{2}+\left(y-y^{\prime}\right)^{2}+\left(z-z^{\prime}\right)^{2}} \rho\left(t, x^{\prime}, y^{\prime}, z^{\prime}\right) d x^{\prime} d y^{\prime} d z^{\prime} .
\end{aligned}
$$

Since smooth solutions $(\rho, u, v, w)$ of $(1.1)-(1.2)$ are considered, the chain rule applies. Taking (1.1) into account, (1.2) becomes

$$
\begin{aligned}
u_{t}+u u_{x}+v u_{y}+w u_{z} & =E_{1}(\rho), \\
v_{t}+u v_{x}+v v_{y}+w v_{z} & =E_{2}(\rho), \\
w_{t}+u w_{x}+v w_{y}+w w_{z} & =E_{3}(\rho),
\end{aligned}
$$

where $u, v$ and $w$ denote the components of the velocity. Physically speaking, the $(u, v, w)$ solution is only of interest when $\rho$ is not equal to 0 . Hence the system of interest is (2.1)-(2.3), together with

$$
\rho_{t}+(\rho u)_{x}+(\rho v)_{y}+(\rho w)_{z}=0,
$$

supplemented with the initial conditions

$$
\rho(t=0)=\rho_{0}, \quad u(t=0)=u_{0}, \quad v(t=0)=v_{0}, \quad w(t=0)=w_{0} .
$$


As we pointed out in the introduction, (2.1)-(2.5) provide a Cauchy problem for the system

$$
U_{t}+A(U) U_{x}+B(U) U_{y}+C(U) U_{z}=\left(\begin{array}{c}
0 \\
E_{1}(\rho) \\
E_{2}(\rho) \\
E_{3}(\rho)
\end{array}\right)
$$

where $U=\left(\begin{array}{c}\rho \\ u \\ v \\ w\end{array}\right)$. This system is not hyperbolic, since for any $\alpha, \beta$ and $\gamma$,

$$
\begin{aligned}
& \alpha A(U)+\beta B(U)+\gamma C(U)= \\
& \quad\left(\begin{array}{cccc}
\alpha u+\beta v+\gamma w & \alpha \rho & \beta \rho & \gamma \rho \\
0 & \alpha u+\beta v+\gamma w & 0 & 0 \\
0 & 0 & \alpha u+\beta v+\gamma w & 0 \\
0 & 0 & 0 & \alpha u+\beta v+\gamma w
\end{array}\right)
\end{aligned}
$$

is not diagonalisable. But as in the classical studies of smooth solutions of hyperbolic systems and bounds of their lifespan (see [4-6]), estimates can be made of the derivatives of the solution. So the bound on $T$ in Theorem 1.1 comes from the limited lifespan of the space derivatives of $(u, v, w)$ which satisfy Riccati-type differential equations. Recall a variant of a classical result on the lifespan and an $L^{\infty}$ bound of a smooth solution to the Riccati equation.

LEMMA 2.1. Let $w$ be the locally defined solution of the system

$$
\left\{\begin{array}{l}
w^{\prime}=a_{0}(t) w^{2}+a_{1}(t) w+a_{2}(t) \\
w(0)=w_{0}
\end{array}\right.
$$

If

$$
\left(\left|w_{0}\right|+\left|a_{2}\right|_{\infty} T e^{\left|a_{1}\right|_{\infty} T}\right)\left|a_{0}\right|_{\infty} T e^{\left|a_{1}\right|_{\infty} T} \leq \frac{1}{2},
$$

then $w$ is defined on $[0, T]$ and

$$
|w|_{\infty} \leq 2\left(\left|w_{0}\right|+\left|a_{2}\right|_{\infty} T e^{\left|a_{1}\right|_{\infty} T}\right)
$$

For the proof of Lemma 2.1, we refer to [11]. The following regularity result on the electric field $E$ will also be needed. 
LEMMA 2.2. Let $\rho$ be a function with compact support $\tilde{K}$, and $1<r<+\infty$. If $\rho \in L^{r}(\tilde{K})$, then for every $(i, j, k)$ such that $0 \leq i+j+k \leq 1, \frac{\partial^{i j j+k} E}{\partial^{i} x \partial^{j} y \partial^{k} z}$ belongs to $L^{r}\left((0, T) \times \mathbb{R}^{3}\right)$ and

$$
\left\|\frac{\partial^{i+j+k} E}{\partial^{i} x \partial^{j} y \partial^{k} z}\right\|_{L^{r}} \leq c_{i j k}\|\rho\|_{L^{r}}
$$

for some constant $c_{i j k}$.

This lemma is a consequence of Calderon-Zygmund inequalities and the theory of singular integrals ([9]).

\section{Proof of Theorem 1.1}

The existence part of Theorem 1.1 is proved by applying Schauder's fixed point theorem to the following map $F$. For every positive number $T$, let $Y_{T}$ be the closed convex subset of $\left(W^{1, \infty}\left((0, T) \times \mathbb{R}^{3}\right)\right)^{3}$ of twice-differentiable functions $(u, v, w)$ with second-order spatial derivatives belonging to $L^{r}\left((0, T) \times \mathbb{R}^{3}\right)$ and

$$
\begin{aligned}
|u|_{\infty} & +\left|u_{x}\right|_{\infty}+\left|u_{y}\right|_{\infty}+\left|u_{z}\right|_{\infty}+\left|u_{x x}\right|_{L^{r}} \\
& +\left|u_{x y}\right|_{L^{r}}+\left|u_{y y}\right|_{L^{\prime}}+\left|u_{x z}\right|_{L^{r}}+\left|u_{y z}\right|_{L^{r}}+\left|u_{z z}\right|_{L^{r}} \leq \frac{1}{T},
\end{aligned}
$$

with analogous bounds for $v$ and $w$. For every $(u, v, w)$ in $Y_{T}$, let $\rho$ be the solution of the Cauchy problem

$$
\rho_{t}+(\rho u)_{x}+(\rho v)_{y}+(\rho w)_{z}=0, \quad \rho(0, x, y, z)=\rho_{0}(x, y, z) .
$$

$F(u, v, w)$ is defined by $F(u, v, w)=(U, V, W)$, where $(U, V, W)$ solves

$$
\begin{aligned}
U_{t}+U U_{x}+V U_{y}+W U_{z} & =E_{1}(\rho), & U(0, x, y, z) & =u_{0}(x, y, z), \\
V_{t}+U V_{x}+V V_{y}+W V_{z} & =E_{2}(\rho), & V(0, x, y, z) & =v_{0}(x, y, z), \\
W_{t}+U W_{x}+V W_{y}+W W_{z} & =E_{3}(\rho), & W(0, x, y, z) & =w_{0}(x, y, z) .
\end{aligned}
$$

$(U, V, W)$ is well defined for $T$ small enough since (3.2)-(3.4) can be expressed as an ordinary differential system with characteristics $(X(t), Y(t), Z(t))$ given by

$$
\begin{aligned}
X^{\prime}(t) & =U(t, X(t), Y(t), Z(t)), \\
Y^{\prime}(t) & =V(t, X(t), Y(t), Z(t)), \\
Z^{\prime}(t) & =W(t, X(t), Y(t), Z(t)) .
\end{aligned}
$$

Here $c, c_{1}, c_{2}, \ldots$ will denote constants independent of $T$ and only depending on the data $\rho_{0}, u_{0}, v_{0}, w_{0}$. If $T$ or some exponential form of $T$ appears in those constants, we bound it from above by 1 and look for an existence time $T$ smaller than 1 .

First, let us study the $\rho$ solution of (3.1) with $(u, v, w)$ in $Y_{T}$. 


\section{LEMMA 3.1. Denote}

$$
\tilde{K}=\{(t, x, y, z) \text { such that } t \in[0,1] \text { and }(x, y, z) \in K+B(0,1)\},
$$

where $B(0,1)$ is the closed unit ball of $\mathbb{R}^{3}$. For any $(u, v, w)$ in $Y_{T}, \rho$ is compactly supported in $\tilde{K}$ and

$$
|\rho|_{\infty}+\left|\rho_{x}\right|_{L^{r}}+\left|\rho_{y}\right|_{L^{r}}+\left|\rho_{z}\right|_{L^{r}} \leq \frac{1}{T}
$$

The estimates in (3.5) follow from the linear ordinary differential equations satisfied by $\rho, \rho_{x}, \rho_{y}, \rho_{z}$ with respect to the derivative $\frac{\partial}{\partial t}+u \frac{\partial}{\partial x}+v \frac{\partial}{\partial y}+w \frac{\partial}{\partial z}$ denoted by $\cdot$. Indeed

$$
\dot{\rho}=-\left(u_{x}+v_{y}+w_{z}\right) \rho, \quad \rho(t=0)=\rho_{0},
$$

so that

$$
\rho(t, x, y, z)=\rho_{0}(\alpha(0), \beta(0), \gamma(0)) e^{-\int_{0}^{\prime}\left(u_{x}+v_{y}+w_{z}\right)(s, \alpha(s), \beta(s), \gamma(s)) d s},
$$

where $(\alpha, \beta, \gamma)$ is defined by

$$
\begin{array}{ll}
\alpha^{\prime}(s)=u(s, \alpha(s), \beta(s), \gamma(s)), & \alpha(t)=x, \\
\beta^{\prime}(s)=v(s, \alpha(s), \beta(s), \gamma(s)), & \beta(t)=y, \\
\gamma^{\prime}(s)=w(s, \alpha(s), \beta(s), \gamma(s)), & \gamma(t)=z .
\end{array}
$$

Hence $\rho$ is supported in $\tilde{K}$. Moreover, the assumptions $\left|u_{x}\right|_{\infty} \leq 1 / T,\left|v_{y}\right|_{\infty} \leq 1 / T$ and $\left|w_{z}\right|_{\infty} \leq 1 / T$ lead to the bound from above of $|\rho|_{\infty}$. The results on $\left|\rho_{x}\right|_{L^{r}},\left|\rho_{y}\right|_{L^{r}}$ and $\left|\rho_{z}\right|_{L^{\prime}}$ are obtained the same way, after noticing that

$$
\begin{aligned}
\left(\begin{array}{l}
\rho_{x} \\
\rho_{y} \\
\rho_{z}
\end{array}\right)= & -\left(\begin{array}{ccc}
u_{x}+v_{y}+w_{z} & v_{x} & w_{x} \\
u_{y} & u_{x}+2 v_{y}+w_{z} & w_{y} \\
u_{z} & v_{z} & u_{x}+v_{y}+2 w_{z}
\end{array}\right)\left(\begin{array}{l}
\rho_{x} \\
\rho_{y} \\
\rho_{z}
\end{array}\right) \\
& -\rho\left(\begin{array}{l}
u_{x x}+v_{x y}+w_{x z} \\
u_{x y}+v_{y y}+w_{y z} \\
u_{x z}+v_{y z}+w_{z z}
\end{array}\right),
\end{aligned}
$$

where the square matrix coefficients and $\rho$ are bounded in $L^{\infty}$, and $u_{x x}+v_{x y}+w_{x z}$, $u_{x y}+v_{y y}+w_{y z}, u_{x z}+v_{y z}+w_{z z}$ are bounded in $L^{r}\left((0, T) \times \mathbb{R}^{3}\right)$.

In order to prove that $\rho$ is bounded in $W^{1, r}(\tilde{K})$, it remains to obtain an $L^{r}(\tilde{K})$ bound for $\rho_{t}$. This comes from an equality derived from (3.1),

$$
\rho_{t}=-\left(u_{x}+v_{y}+w_{z}\right) \rho-u \rho_{x}-v \rho_{y}-w \rho_{z},
$$


and the previous $L^{r}(\tilde{K})$ bounds of $\rho, \rho_{x}, \rho_{y}, \rho_{z}$ as well as the $L^{\infty}(\tilde{K})$ bounds of $u, v$, $w, \boldsymbol{u}_{x}, v_{y}$ and $w_{z}$.

Let us prove that $F$ maps $Y_{T}$ into $Y_{T}$. Thanks to the results of Lemmas 2.1 and 2.2, we may use the same proof as in Lemma 3.1 to show that

$$
|U|_{\infty} \leq \frac{1}{2 T}, \quad|V|_{\infty} \leq \frac{1}{2 T}, \quad|W|_{\infty} \leq \frac{1}{2 T}
$$

for $T$ small enough. If ' denotes the derivative $\frac{\partial}{\partial t}+U \frac{\partial}{\partial x}+V \frac{\partial}{\partial y}+W \frac{\partial}{\partial z}$, the firstorder spatial derivatives of $U, V$ and $W$, denoted by $X$, are solutions of a differential equation,

$$
X^{\prime}=G_{1}(X)+G_{2}\left(E_{x}, E_{y}, E_{z}\right)
$$

with $G_{1}$ independent of $\rho$ and $G_{2}$ linear. Since $\operatorname{div} E_{x}=\rho_{x}, \operatorname{div} E_{y}=\rho_{y}, \operatorname{div} E_{z}=\rho_{z}$ and $r>3$, Lemmas 2.2 and 3.1 imply that $X$ is bounded for $T$ small enough. In order to complete the proof of the inclusion of $F\left(Y_{T}\right)$ in $Y_{T}$, it remains to prove that the bounds of the second-order spatial derivatives of $U, V$ and $W$ hold. Denote by $Y$ and $Z$ the vectors of the second-order spatial derivatives of $(U, V, W)$ and $E$ respectively. $Y$ satisfies a linear differential system,

$$
Y^{\prime}=M Y+Z,
$$

where $M$ is a $6 \times 6$ matrix whose coefficients are bounded in $L^{\infty}$ as linear combinations of the first spatial derivatives of $U, V$ and $W$. Moreover, $Y(t=0)$ belongs to $L^{r}$ as the second-order derivatives of $\left(u_{0}, v_{0}, w_{0}\right)$ must belong to $L^{r}$. Hence $Y$ is bounded in $L^{r}$ by $L^{r}$ bounds of $Z$ that follow from Lemma 2.2 .

Let us now prove the continuity of $F$ for the $\left(W^{1, \infty}\left([0, T] \times \mathbb{R}^{3}\right)\right)^{3}$ topology. We first observe that $(U, V, W)$ continuously depends on $(u, v, w)$. If $(\tilde{u}, \tilde{v}, \tilde{w})$ is given in $Y_{T},(\tilde{U}, \tilde{V}, \tilde{W})=F(\tilde{u}, \tilde{v}, \tilde{w})$ and ' denotes the derivative $\frac{\partial}{\partial t}+\tilde{U} \frac{\partial}{\partial x}+\tilde{V} \frac{\partial}{\partial y}+\tilde{W} \frac{\partial}{\partial z}$, then for every $(U, V, W)=F(u, v, w)$,

$$
(U-\tilde{U})^{\prime}+(U-\tilde{U}) U_{x}+(V-\tilde{V}) U_{y}+(W-\tilde{W}) U_{z}=E_{1}(\rho-\tilde{\rho}),
$$

and similar equations hold for $V-\tilde{V}$ and $W-\tilde{W}$. When $(u, v, w)$ tends to $(\tilde{u}, \tilde{v}, \tilde{w})$ in $\left(W^{1, \infty}\left([0, T] \times \mathbb{R}^{3}\right)\right)^{3}, \rho$ tends to $\tilde{\rho}$ in $L^{\infty}(\tilde{K})$, so that the $L^{\infty}\left([0, T] \times \mathbb{R}^{3}\right)$ convergence of $E_{i}(\rho-\tilde{\rho}), 1 \leq i \leq 3$ towards 0 is straightforward. Then the boundedness of the first spatial derivatives of $(U, V, W)$ due to the fact that $(U, V, W)$ belongs to $Y_{T}$ implies the $L^{\infty}$-continuity of $\mathrm{F}$.

Then the first spatial derivatives of $(U, V, W)$ continuously depend on $(u, v, w)$ and its first spatial derivatives in the $L^{\infty}$-topology. Indeed $\rho-\tilde{\rho}$ is compactly supported and tends to 0 in $L^{r}$, so that Lemma 2.2 implies that $E_{i}(\rho-\tilde{\rho}), 1 \leq i \leq 3$ tend to 0 in 
$W^{2, r}$. Then the Rellich-Kondrakov continuous imbedding of $W^{1, r}\left([0, T] \times \mathbb{R}^{3}\right)$ into $L^{\infty}\left([0, T] \times \mathbb{R}^{3}\right)$ implies that $\partial_{x} E_{i}(\rho-\tilde{\rho}), \partial_{y} E_{i}(\rho-\tilde{\rho})$ and $\partial_{z} E_{i}(\rho-\tilde{\rho}), 1 \leq i \leq 3$ tend to 0 in $L^{\infty}\left([0, T] \times \mathbb{R}^{3}\right)$ when $(u, v, w)$ tends to $(\tilde{u}, \tilde{v}, \tilde{w})$ in $\left(W^{1, \infty}\left([0, T] \times \mathbb{R}^{3}\right)\right)^{3}$. Finally

$$
\begin{aligned}
U_{t} & =-U U_{x}-V U_{y}-W U_{z}+E_{1}(\rho), \\
V_{t} & =-U V_{x}-V V_{y}-W V_{z}+E_{2}(\rho), \\
W_{t} & =-U W_{x}-V W_{y}-W W_{z}+E_{3}(\rho),
\end{aligned}
$$

so that $U_{t}, V_{t}$ and $W_{t}$ continuously depend on $(u, v, w)$.

It remains to show that $F$ is a compact map, in order to apply the Schauder fixed point theorem. Let $\left(u^{n}, v^{n}, w^{n}\right)$ be a sequence of $Y_{T}$ bounded for the $\left(W^{1, \infty}\left([0, T] \times \mathbb{R}^{3}\right)\right)^{3}$ norm. Thanks to Lemma 3.1, the sequence $\left(\rho^{n}\right)$ associated with $\left(u^{n}, v^{n}, w^{n}\right)$ by (3.1) is bounded in $W^{1, r}(\tilde{K})$. Then the Rellich-Kondrakov compact imbedding of $W^{1, r}(\tilde{K})$ into $L^{r}(\tilde{K})$ for $r>3$ allows us to conclude that $\left(\rho^{n}\right)$ is relatively compact in $L^{r}(\tilde{K})$. Thus arguments similar to those used for proving the continuity of $F$ may be used to prove that $F\left(u^{n}, v^{n}, w^{n}\right)$ is relatively compact in $\left(W^{1, \infty}\left([0, T] \times \mathbb{R}^{3}\right)\right)^{3}$.

Hence Schauder's fixed point theorem establishes the existence of a solution of the Cauchy problem (2.1)-(2.5).

Finally, let $(\rho, u, v, w)$ and $(\tilde{\rho}, \tilde{u}, \tilde{v}, \tilde{w})$ be two solutions to $(2.1)-(2.5)$ such that the second-order spatial derivatives of $u, v, w, \tilde{u}, \tilde{v}, \tilde{w}$ belong to $L^{r}\left([0, T] \times \mathbb{R}^{3}\right)$. The first spatial derivatives of $u-\tilde{u}, v-\tilde{v}$ and $w-\tilde{w}$ can be expressed in terms of the second-order spatial derivatives of $u, v, w$ and convolution products between $\rho$ and the first-order derivatives of the kernel arising in the solution of the Poisson equation in $\mathbb{R}^{3}$. A contraction argument for $(\rho-\tilde{\rho}, u-\tilde{u}, v-\tilde{v}, w-\tilde{w})$ and the first-order spatial derivatives of $(u-\tilde{u}, v-\tilde{v}, w-\tilde{w})$ in $L^{2} \times\left(L^{\frac{2 r}{r-2}}\right)^{3}$ and $\left(L^{2}\right)^{9}$ respectively can be used in order to prove that $\rho=\tilde{\rho}, u=\tilde{u}, v=\tilde{v}$ and $w=\tilde{w}$. This proves the uniqueness result.

\section{References}

[1] F. Bouchut, "On zero pressure gas dynamics", in Advances in kinetic theory and computing selected papers, Series on Advances in Mathematics for Applied Sciences 22, (World Scientific, Singapore, 1994) 171-190.

[2] Y. Brenier, E. Grenier, "Sticky particles and scalar conservation laws", SIAM J. Num. Anal. 35 (1998) 2317-2328.

[3] A. Forestier and P. Le Floch, "Multivalued solutions to some non-linear and non-strictly hyperbolic systems", Japan J. Indust. Appl. Math. 9 (1992) 1-23.

[4] P. Hartman and A. Wintner, "On hyperbolic partial differential equations", Amer. J. Math. 74 (1952) 834-864. 
[5] L. Hörmander, "The lifespan of classical solutions of non-linear hyperbolic equations", 1985, Report no 5.

[6] P. Lax, "Hyperbolic systems of conservation laws", Comm. Pure Appl. Math. 10 (1957) 537-566.

[7] A. Majda, Compressible fluid flow and systems of conservation laws in several space variables (Springer, 1984).

[8] A. Nouri, "Paraxial approximation of Vlasov-Maxwell system: Laminar beams", M3AS 4 (1994) 203-221.

[9] E. M. Stein, Singular integrals and differentiability properties of functions (Princeton University Press, 1970).

[10] D. H. Wagner, "Equivalence of the Euler and Lagrangian equations of gas dynamics for weak solutions", J. Differential Eqns. 68 (1987) 118-136.

[11] H. F. Weinberger and M. H. Protter, Maximum principles in differential equations (Prentice-Hall, Inc., 1967). 